\title{
Mean glandular dose values used for the mammography screening program in Poland according to the type of image registration system
}

\author{
Ewa Fabiszewska, Katarzyna Pasicz, Iwona Grabska
}

Between 2007-2014, there have been considerable changes in the use of different types of detector systems at mammography facilities undertaking screening programs in Poland. The use of screen-film systems (termed SF systems) has largely decreased and been replaced by either computed radiography (termed CR systems) or digital radiography systems (termed DR systems); this inevitably affecting mean glandular dose values. The study aim was to evaluate changes of mean glandular dose values for a dose exposure of $4.5 \mathrm{~cm}\left(\mathrm{MGD}_{4,5 \mathrm{cmPMMA}}\right)$ achieved by using different image detectors. The study consisted of analysing 1499 protocols for quality control tests carried out by medical physicists at 16 Regional Coordination Offices in 2007, 2011, 2012 and 2013. The mean CR system values were higher than for SF systems; by $25 \%$ in 2011 , by $26 \%$ in 2012 , and by $28 \%$ in 2013 . In subsequent years, the MGD $_{4,5 \mathrm{cmPMMA}}$ mean values for DR systems were higher than for SF systems by respectively $15 \%, 4 \%$ and $5 \%$. Also in the subsequent years, the $M_{4,5 \mathrm{cmPMMA}}$ mean values for CR systems were higher than for DR systems by respectively $13 \%, 23 \%$ and $24 \%$. In all cases, the $\mathrm{MGD}_{4,5 \mathrm{cmPMMA}}$ mean values were within the acceptable level of $2.5 \mathrm{mGy}$. The $\mathrm{MGD}_{4,5 \mathrm{cmPMMA}}$ mean values were different, depending on the detection system used for mammography screening in Poland.

NOWOTWORY J Oncol 2017; 67, 2: 115-120

Key words: mean glandular dose, mammography screening, quality control

\section{Introduction}

In Poland, the 'Polish National Breast Cancer Early Detection Program for Women aged from 50 to 69 ' has been undertaken since 2006 where such women underwent mammography examination. Each woman was examined every second year. Until 2015, the program was managed by a Central Coordination Office at the Maria SkłodowskaCurie Memorial Centre and Institute of Oncology in Warsaw together with 16 Regional Coordination Offices in each region (voivodeship) in Poland [1]. The coordination offices were chiefly responsible for assuring the quality of mammography screening examinations. For this purpose medical physicists at each mammography facility carried out quality control tests of mammography equipment consisting of: X-ray beam geometry, breast compression, spatial resolution, threshold contrast, artefacts, compensation of phantom thickness changes and high voltage values gen- erated by the Automatic Exposure Control (AEC) systems, routine exposure times and the conditions and quality of the mammography image display. Moreover, the mean glandular dose values for an exposure of a $4.5 \mathrm{~cm}$ thick phantom were determined (later called $\mathrm{MGD}_{4,5 \mathrm{cmPMMA}}$ ). Between 2007-2008 and between 2011-2015, all the mammography facilities carrying out screening examinations in Poland were quality controlled. During 2009 and 2010 however, quality control was performed for only those mammography facilities which had the worst outcomes in the previous year as well as those, which in any given year, had been part of the screening programs. In 2009, 200/340 mammography units (about 59\%) were quality assessed, and in 2010, 271/ /325 (about 83\%).

Over 2007-2009 screening was either performed solely for those mammography units with film detector systems (termed screen-film systems - SF) or with digital detectors 
(termed digital radiography systems - DR). Since 2010 and according to President of the National Health Fund regulations, systems with an imaging plate (made of photosimulable phosphor, termed computed radiography systems $-\mathrm{CR}$ ) have been included in the screening program. Within recent years, the SF systems have been gradually replaced by $C R$ and DR systems, which thereby must have affected the radiation doses received by the women examined. The study aim was to evaluate changes in the $M_{4,5 \mathrm{cmPMMA}}$ doses when using different image detectors.

\section{Materials and methods}

The study consisted of analysing 1499 protocols of quality control tests carried out by medical physicists at 16 Regional Coordination Offices in 2007, 2011, 2012 and 2013. The quality control covered those parameters of mammography systems responsible for image quality and $M_{4,5 \mathrm{cmPMMA}}$ of which the outcomes from 2007 and 2011 were published in the Nowotwory Journal of Oncology [2]. For this study only the $\mathrm{MGD}_{4,5 \mathrm{cmPMMA}}$ dose values for the $4.5 \mathrm{~cm}$ thick PMMA phantom were analysed. Additionally, on the basis of the above mentioned protocols, the numbers of mammography units with particular detector types SF, CR, and DR, were established. The numbers of mammography units taking part in the screening program for 2014 were obtained from the Central Coordination Office report [3].

\section{Determination of $M G D_{4,5 \mathrm{~cm} P M M A}$}

Medical physicists from each Regional Coordination Office measured $M G D_{4,5 \mathrm{cmPMMA}}$ doses according to the methods published in 'Additional factors for the estimation of mean glandular breast dose using the UK mammography dosimetry protocol' [4], and to European recommendations $[5,6]$.

The $M G D_{4,5 \mathrm{cmPMMA}}$ values were calculated by the formula:

$$
\mathrm{MGD}_{4,5 \mathrm{cmPMMA}}=\mathrm{K} \cdot \mathrm{g} \cdot \mathrm{c} \cdot \mathrm{S}
$$

where:

$\mathrm{MGD}_{4,5 \mathrm{cmPMMA}}$ - mean glandular dose for $4.5 \mathrm{~cm}$ thick PMMA phantom;

$\mathrm{K}$ - entrance surface air kerma (without backscatter) at the upper surface of the breast;

g, c, s - conversion factors determined by Dance et al. [4] using Monte Carlo techniques.

The measurements were performed at parameter settings used routinely for mammography examinations. The $M_{4,5 \mathrm{cmPMMA}}$ values were compared to the limit values in the European recommendations [5, 6]. For this study, the $\mathrm{MGD}_{4,5 \mathrm{cmPMMA}}$ values determined in 2007 and 2011-2013 were analysed.

\section{Data analysis}

Using the 2011-2014 data, the proportional use of the various systems employed in Poland were determined. Based on the number of mammography units quality controlled in a given year, a linear projection trend of SF systems being replaced by the $C R$ and $D R$ systems was determined. Thus the trend enabled the year to be established at which the last SF system is to disappear; assuming a continuing like trend in the future. For each year, the mean, standard deviation and minimum and maximum $\mathrm{MGD}_{4,5 \mathrm{cmPMMA}}$ values for all the mammography systems were calculated. The mammography systems were divided into three groups: SF, $C R$ and DR where likewise the means, standard deviations, and minimum and maximum values of $M_{4,5 \mathrm{cmPMMA}}$ were calculated. Additionally, the mean value of $\mathrm{MGD}_{4,5 \mathrm{cmPMMA}}$ in the year when all SF systems would be replaced, was calculated.

To allow comparison of $\mathrm{MGD}_{4,5 \mathrm{cmPMMA}}$ values for different systems, outcomes per year were divided into three groups consisting of firstly SF and CR systems, secondly CR and DR systems and thirdly the SF and DR systems. For statistical analysis a baseline assumption was made that the mean value for each group was the same. For evaluating the variance differences between different groups, a F-Snedecor test was used. A p $<0.05$ was considered statistically significant. After establishing that the variances in all groups were the same, the t-student test was performed at the level of $\alpha=0.05$. In cases where the variances in both analysed groups were different, a statistical Cochran-Crox test was used.

\section{Results and discussion}

The number of mammography systems used during the 5 -year study period increased by $32 \%$ compared to the number of systems starting in 2007 which was 298. During 2011-2014, the number of mammography units was about 400 and remained at the same level (Tab. I). Only the proportions of the different system types changed. In Figure 1, the proportions of SF, CR, and DR systems used for the screening program in Poland, in 2007 and 2011-2014 are presented. Over time the number of SF systems has diminished compared to the CR and DR systems. In 2007, SFs constituted $97 \%$ of operating systems but in 2014 this decreased to $56 \%$. The proportion of CR systems in 2007 was only $1 \%$, but by the end of 2014 this rose to $29 \%$. In 2007 the proportion of DR systems was only $2 \%$ but rose to $15 \%$ in 2014. There were almost twice more CR systems than DR systems in 2014 due to the former's lower price and easier adaptation of existing systems to $C R$ rather than to $D R$ systems. Assuming that the trend of replacing SF systems with CR and DR systems continues, SF systems will be absent from mammography screening in Poland by 2022 (Fig. 2). The CR to DR system ratio would at such time be 2.5 , thus favouring a large advantage of CR over DR systems. 
Table I. MGD $4,5 \mathrm{cmPMMA}$ mean values, standard deviations, minimum and maximum dose values for all mammography system types for 2007 and $2011-2013$

\begin{tabular}{lccccc}
\hline \multicolumn{1}{c}{} & \multicolumn{3}{c}{$\mathrm{MGD}_{4,5 \mathrm{cmPMMA}}[\mathrm{mGy}]$} \\
\hline Year & $\begin{array}{c}\text { Number of } \\
\text { mammography units }\end{array}$ & Mean value & Standard deviation & Minimum value & Maximum value \\
\hline 2007 & 299 & 1.94 & 0.72 & 0.55 & 4.76 \\
2011 & 391 & 1.49 & 0.43 & 0.70 & 6.63 \\
2012 & 405 & 1.42 & 0.36 & 0.53 & 3.20 \\
2013 & 400 & 1.48 & 0.39 & 0.30 & 2.50 \\
\hline
\end{tabular}

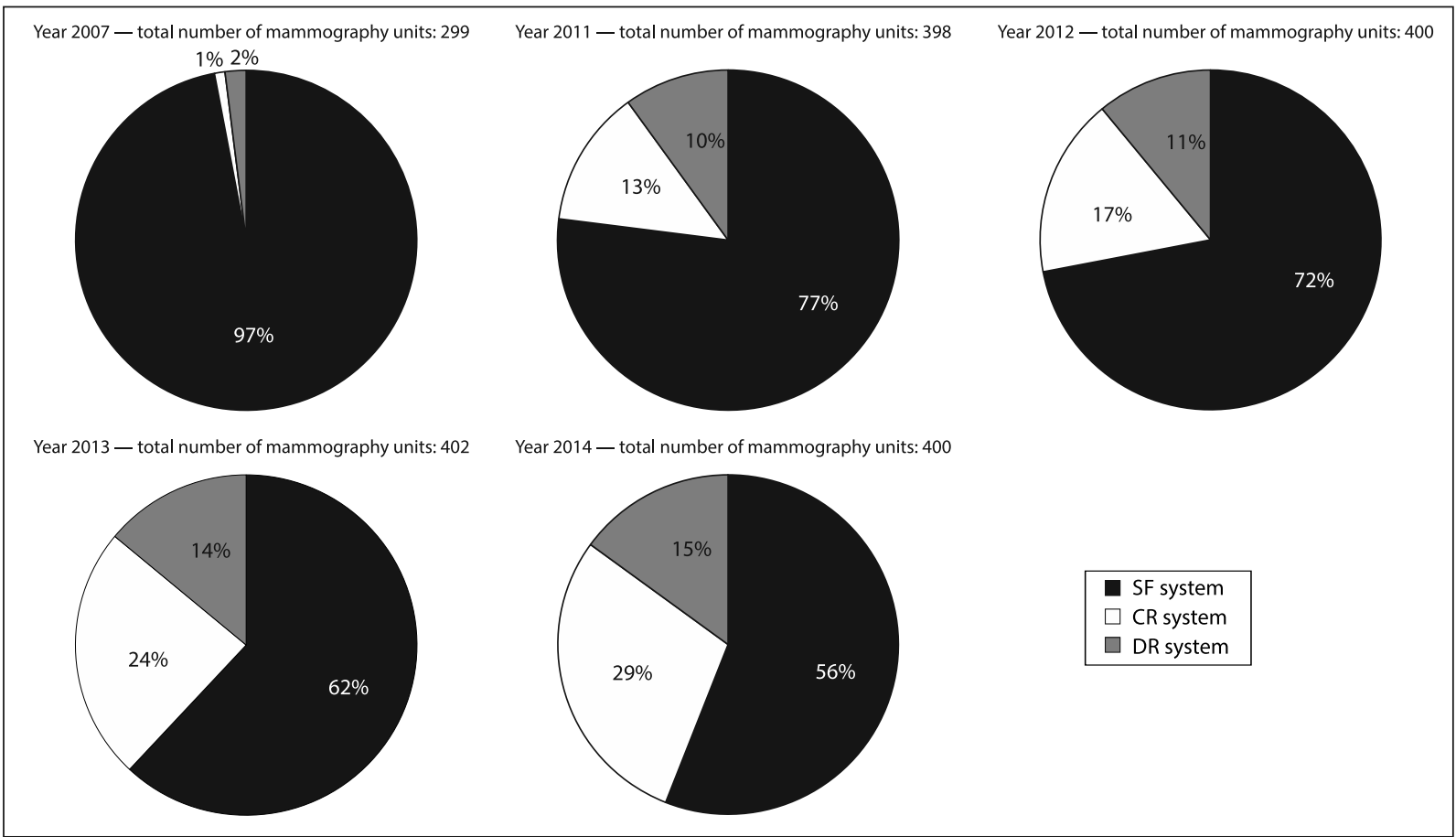

Figure 1. Proportions of different mammography systems for the screening program in Poland in 2007, 2011, 2012, 2013 and 2014

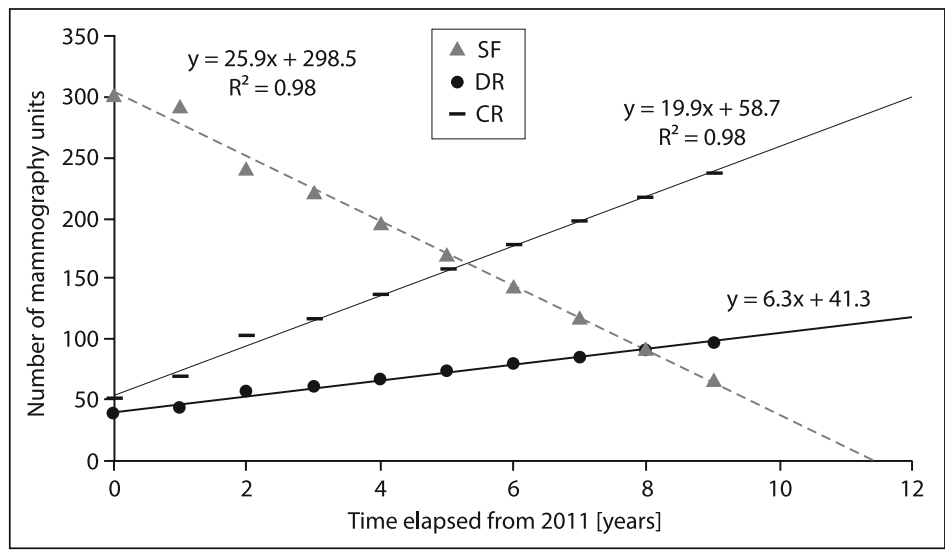

Figure 2. Actual and forecasted (2015-2013) numbers of SF, CR, and DR systems used for the mammography screening program in Poland 


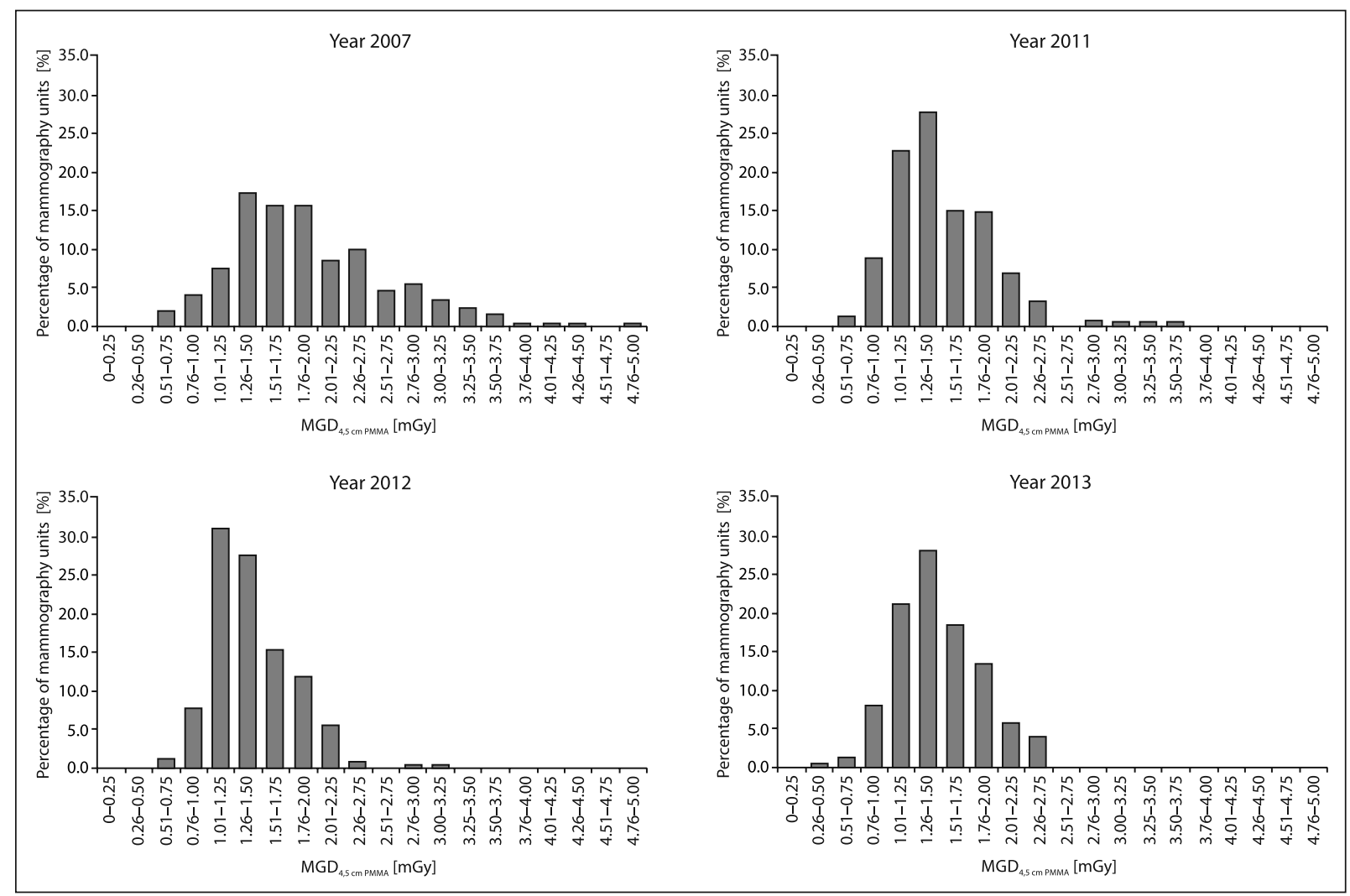

Figure 3. Histograms of $\mathrm{MGD}_{4,5 \mathrm{cmPMMA}}$ values for all mammography systems used in Poland for 2007, 2011, 2012, and 2013

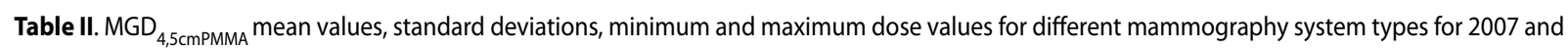
2011-2013

\begin{tabular}{|c|c|c|c|c|c|c|}
\hline \multicolumn{7}{|c|}{$\mathrm{MGD}_{4.5 \mathrm{cmPMMA}}[\mathrm{mGy}]$} \\
\hline Year & Type of detector & $\begin{array}{c}\text { Number of } \\
\text { mammography units }\end{array}$ & Mean value & Standard deviation & Minimum value & Maximum value \\
\hline \multirow[t]{3}{*}{2011} & SF & 302 & 1.41 & 0.37 & 0.74 & 3.63 \\
\hline & $\mathrm{CR}$ & 51 & 1.89 & 0.52 & 0.70 & 3.40 \\
\hline & DR & 38 & 1.65 & 0.43 & 0.80 & 2.30 \\
\hline \multirow[t]{3}{*}{2012} & SF & 293 & 1.33 & 0.29 & 0.68 & 2.29 \\
\hline & $\mathrm{CR}$ & 69 & 1.79 & 0.37 & 1.00 & 3.20 \\
\hline & DR & 43 & 1.38 & 0.42 & 0.53 & 2.80 \\
\hline \multirow[t]{3}{*}{2013} & SF & 241 & 1.35 & 0.29 & 0.63 & 2.15 \\
\hline & $\mathrm{CR}$ & 103 & 1.88 & 0.35 & 1.00 & 2.50 \\
\hline & DR & 56 & 1.42 & 0.32 & 0.90 & 2.30 \\
\hline
\end{tabular}

Histograms of $\mathrm{MGD}_{4,5 \mathrm{cmPMMA}}$ values from 2007, 2011, 2012 and 2013 indicate a consecutive dose decrease (Fig. 3) observed for all the three mammography systems. The scale on the $x$-axis is the same for all histograms so that differences in $\mathrm{MGD}_{4,5 \mathrm{cmPMMA}}$ distributions can be demonstrated between years. When comparing $\mathrm{MGD}_{4,5 \mathrm{cmPMMA}}$ values for all systems in 2007 to 2013, then the mean value in 2013 was $1.48 \mathrm{mGy}$, which is about 24\% lower than that in 2007 (Tab. I).
In Table I, the $\mathrm{MGD}_{4,5 \mathrm{cmpMmA}}$ mean values, standard deviations, minimum and maximum values for all system types in 2007 and 2011-2013 are presented. Comparing the $\mathrm{MGD}_{4,5 \mathrm{cmPMMA}}$ mean values between the various system types (Tab. II) indicates that those for CR systems were higher than for SF systems: by $25 \%$ in 2011 , by $26 \%$ in 2012 , and by $28 \%$ in 2013. The $\mathrm{MGD}_{4,5 \mathrm{cmPMMA}}$ mean values for $\mathrm{DR}$ systems were higher than for SF systems in subsequent years by 
respectively $15 \%, 4 \%$ and $5 \%$ whereas the $M_{4} D_{4,5 \mathrm{cmPMMA}}$ mean values for $C R$ systems were higher than for $D R$ systems in subsequent years by respectively $13 \%, 23 \%$ and $24 \%$. In all cases, the $\mathrm{MGD}_{4,5 \mathrm{~cm} P M M A}$ mean values were within the acceptable level of $2.5 \mathrm{mGy}$. When analyzing individual cases it was found that MGD values in 2011 exceeded the acceptable level in 14 SF systems and 1 CR system, whilst in 2012 this occurred only in 1 CR system. In 2013, MGD values did not exceed the $2.5 \mathrm{mGy}$ at any time. A comparison between those system 2011 and 2013 results exceeding the acceptable level revealed that $1 \mathrm{SF}$ system and $1 \mathrm{CR}$ system had been taken out of service, however the exposure conditions for remaining CR systems were changed so that MGD values stayed within the acceptable limit. Nevertheless, the maximum values for $C R$ systems were $16.8 \%$ higher than for SF systems and $8.7 \%$ higher than for DR systems, whilst the DR values were $7 \%$ higher than for SF systems.

According to the European recommendations $[5,6]$, every mammography facility should endeavour to keep values of the mean glandular dose (termed MGD) at an achievable level of $2 \mathrm{mGy}$. In the literature, $\mathrm{MGD}_{4,5 \mathrm{cmPMMA}}$ values for DR systems are stated not to exceed $2.0 \mathrm{mGy}$ per exposure [7-11]. Our data indicate that in 2011 the achievable level was exceeded by 15 SF systems ( $5 \%), 18 \mathrm{CR}$ systems ( 35\%), and 10 DR systems ( 10\%). In 2012, the achievable level was exceeded by 9 SF systems ( 3\%), 15 CR systems ( 22\%), and 3 DR systems ( 7\%) whereas in 2013, the achievable level was exceeded for 7 SF systems ( 3\%), 30 CR systems ( 29\%), and 2 DR systems ( 4\%).

Statistically significant differences in $\mathrm{MGD}_{4,5 \mathrm{~cm} P M M A}$ mean values were found between SF and CR systems and between DR and CR systems, however such differences were insignificant between SF and DR systems. This means that during 2011-2013, the $M_{4,5 \mathrm{cmPMMA}}$ mean values for $C R$ systems were significantly different from the $\mathrm{MGD}_{4,5 \mathrm{cmPMMA}}$ mean values for both SF and DR systems. Literature data indicate that mean MGD values for DR systems are about $32 \%$ lower than for SF systems, and those for CR systems are close to the values for SF systems [12]. Young et al. showed that MGD values for DR systems were lower by $25 \%$ than those for SF systems [13]. Similar results have been shown by Hendrik et al. [14].

MGD values presented in this paper clearly differ from those in the literature but it is difficult to definitively account for such discrepancies. Nevertheless, our results indicate that it is possible to decrease $X$ ray doses in the mammography screening in Poland, especially for CR systems. Most likely this would require optimising the exposure parameters taking into account the image quality, and perhaps even individually modernising the mammography equipment for every system. In an earlier study [15], the present authors described an attempt to optimize exposure parameters for increasing the MGD value and to maintain image quality.
Although such attempts were directed to the DR systems, they may also be applied to CR systems.

Assuming that the the number of mammography units until 2022 grows at the same rate as during 2011-2014, then the $\mathrm{MGD}_{4,5 \mathrm{cmPMMA}}$ mean value in 2022 was estimated to be $1.66 \mathrm{mGy}$. Such a $\mathrm{MGD}_{4,5 \mathrm{cmPMMA}}$ mean value would in fact be about $12 \%$ larger than the $\mathrm{MGD}_{4,5 \mathrm{cmPMMA}}$ mean value for all mammography systems in Poland in 2013.

\section{Conclusions}

$\mathrm{MGD}_{4,5 \mathrm{~cm} P M M A}$ mean values differ according to the detection system used for mammography screening in Poland. For CR systems they are significantly higher than for SF and DR systems. In order to adhere to the ALARA principle, it appears necessary to make every effort to decrease current $M G D_{4,5 \mathrm{cmPMMA}}$ mean values, especially for CR systems.

\section{Acknowledgments}

The authors are grateful to the Central Coordination Office for providing access to protocols of quality control tests undertaken during auditing at mammography screening facilities in 2007, 2011-2013.

\section{Ewa Fabiszewska, MD, PhD}

Department of Medical Physics

Maria Skłodowska-Curie Memorial Cancer

Centre and Institute of Oncology

W.K. Roentgena 5

02-781 Warszawa, Poland

e-mail:e.fabiszewska@zfm.coi.pl

Received: 16 Nov 2016

Accepted: 9 Mar 2017

\section{References}

1. Fabiszewska E, Jankowska K, Grabska I et al. Individual doses for women undergoing screening mammography examinations in Poland in 2007. J Radiol Prot 2011; 31: 467-475.

2. Fabiszewska E, Grabska I, Pasicz K et al. Assessment of mammography equipment quality in mammography screening facilities in 2007 and 2011 in Poland. Nowotwory J Oncol 2014; 64: 119-128.

3. The report of the quality control auditis in the frame "Polish National Breast Cancer Early Detection Program" in mammography facilities taking part in in the "Breast cancer profilactic programme" in 2014. http://www.profilaktykaraka.coi.waw.pl.

4. Dance DR, Skinner CL, Young KC et al. Additional factors for the estimation of mean glandular breast dose using the UK mammography dosimetry protocol. Phys Med Biol 2000; 45: 3225-3240.

5. Perry N, Broeders M, de Wolf $\mathrm{C}$ et al. European guidelines for quality assurance in breast cancer screening and diagnosis. $4^{\text {th }}$ ed. Luxemburg: Office for the Official Publications of the Europen Communities, 2006.

6. Perry N, Broeders M, de Wolf C et al. (eds.). European guidelines for quality assurance in breast cancer screening and diagnosis. $4^{\text {th }}$ ed. Luxemburg: Office for Official Publications of the European Union, 2013.

7. Hermann KP, Obenauer S, Marten K et al. Average glandular dose with amorphous silicon full-field digital mammography - Clinical results. Rofo 2002; 174: 696-699.

8. Gosch D, Jendrass $S$, Scholz $M$ et al. Radiation exposure in full-field digital mammography with a selenium flat-panel detector. Rofo 2006; 178: 693-697. 
9. Gennaro G, di Magio C. Dose comparison between screen/film and full-field digital mammography. Eur J Radiol 2006; 16: 2559-2566.

10. Fischmann A, Siegmann KC, Wersebe A et al. Comparison of full-field digital mammography and film-screen mammography: image quality and lesion detection. Br J Radiol 2005; 78: 312-315.

11. Gennaro G, Baldelli $P$, Taibi A et al. Patient dose in full-field digital mammography: an Italian survey. Eur J Radiol 2004; 14: 645-652.

12. Oduko JM, Young KC, Burch A. A survey of patient doses from digital mammography systems in the UK in 2007 to $2009,10^{\text {th }}$ International Workshop, IWDM 2010, Girona, Catalonia, Spain, June 16-18, 2010, Proceedings.
13. Young KC, Oduko JM. Radiation doses received in the United Kingdom breast screening programme in 2010 to 2012. Br J Radiol 2016; 89: 20150831.doi: 10.1259/brj.20150831.

14. Hendrick RE, Pisano ED, Averbukh A et al. Comparison of acquisition parameters and breast dose in digital mammography and screen-film mammography in the American College of Radiology Imaging Network digital mammographic imaging screening trial. AJR Am J Roentgenol 2010; 194: 362-369.

15. Uchyiyama N, Zanachetta do Nascimento M (eds.). Mammography recent advences. InTech, 2012. doi: 10.5772/2508. 